\title{
14 Utopie und Dystopie als Kontrafaktik
}

Kaum ein anderes Genre der politischen Kunst erfreut sich in der Gegenwart insbesondere im Bereich der Populärkultur - vergleichbar großer Popularität wie die Dystopie: Man denke nur an Werke wie Suzanne Collins' Hunger GamesTrilogie (2008, 2009, 2010), an Juli Zehs dystopische Romane Corpus Delicti (2009) und Leere Herzen (2017), an Filme wie Alfonso Cuaróns Children of Men (2006), an die Matrix-Trilogie (1999, 2003) oder V for Vendetta (2005) der Wachowski-Geschwister, an populäre Serien wie Black Mirror (seit 2011) oder The Handmaid's Tale (seit 2017, basierend auf Margaret Atwoods gleichnamigem Roman aus dem Jahre 1985), an Karen Duves skurrile Vision eines Staatsfeminismus im Roman Macht (2016), an Marc-Uwe Klings Big-Data-kritische „Zukunftssatire“1031 QualityLand (2017) oder an Michel Houellebecqs kontrovers diskutierte Vision einer Islamisierung Frankreichs im Roman Unterwerfung (2015). Anders als in anderen amimetischen Genres wie Fantasy oder Science-Fiction, mit denen die Dystopie häufig Hybridbildungen eingeht ${ }^{1032}$, ist für das Genre der Utopie und Dystopie eine politische Dimension konstitutiv: Während Werke der Fantasy oder Science-Fiction nicht notwendigerweise einen politisch-kritischen Gegenwartskommentar mitumfassen, lassen sich fiktionale Werke nicht sinnvollerweise als utopisch oder dystopisch bezeichnen, ohne damit zugleich die Möglichkeit einer politischen Lesart dieser Werke nahezulegen. Durch den Entwurf einer räumlich entrückten oder zukünftigen, in jedem Falle aber fiktiven Gesellschaft formulieren utopische und dystopische Texte eine Kritik der Gegenwart, indem sie etwa aktuelle Gesellschaftstendenzen satirisch überzeichnen und damit zu einem normativ geladenen Vergleich zwischen der fiktiven Gesellschaft innerhalb der Diegese und der realen Gesellschaft der Leser auffordern. Stilbildend für das Genre haben im 20. Jahrhundert insbesondere die Romane Brave New World (1932) von Aldous Huxleys und 1984 (1949) von George Orwells gewirkt. ${ }^{1033}$ In vielen der nachfolgenden dystopischen Romane, Filme oder Comics - inklusive den im Folgenden zu diskutierenden Texten von Juli Zeh und Leif Randt - lassen sich Bezüge auf diese Klassiker des Genres identifizieren.

1031 Kling: QualityLand, Klappentext, Buchdeckel vorne, Innenseite.

1032 Vgl. Hans-Edwin Friedrich: Science-Fiction. In: Dieter Lamping (Hg.): Handbuch der literarischen Gattungen. Stuttgart 2009, S. 672-677, hier S. 674; Hans Esselborn: Vorwort. In: Ders. (Hg.): Utopie, Antiutopie und Science Fiction im deutschsprachigen Roman des 20. Jahrhunderts. Würzburg 2003, S. 7-11, hier S. 9-11.

1033 Vgl. Elena Zeißler: Dunkle Welten. Die Dystopie auf dem Weg ins 21. Jahrhundert. Marburg 2008, S. 37-56; Wilhelm Voßkamp: Utopie. In: Dieter Lamping (Hg.): Handbuch der literarischen Gattungen. Stuttgart 2009, S. 740-750, hier S. $748 \mathrm{f}$.

Ә Open Access. (c) 2022 Michael Navratil, publiziert von de Gruyter. (c) BY-NC-ND Dieses Werk ist lizenziert unter einer Creative Commons Namensnennung - Nicht-kommerziell - Keine Bearbeitung 4.0 International Lizenz. https://doi.org/10.1515/9783110763119-017 
Utopie und Dystopie werden im Folgenden gemeinsam verhandelt. Der Unterschied zwischen beiden besteht vor allem in ihrer gegensätzlichen Wertungstendenz: Bei der Utopie handelt es sich um einen eher positiven, bei der Dystopie hingegen um einen eher negativen Gesellschaftsentwurf. ${ }^{1034}$ Mit Blick auf die Gegenwartsliteratur ist dabei zu bemerken, dass auch tendenziell positive Gesellschaftsentwürfe eine starke Neigung zum normativen Umschlag aufweisen, dass sich also auch vordergründige Utopien schlussendlich meist als Dystopien entpuppen oder zumindest Elemente enthalten, die - je nach Lesart - eine entschieden negative Deutung erlauben. Angesichts der „inhärente[n] Dialektik von utopischer Hoffnung und dystopischem Pessimismus“1035, wie sie gerade in der Gegenwartskunst vorherrscht, erscheint eine gemeinsame Verhandlung der beiden Genrevarianten umso sinnvoller. ${ }^{1036}$

1034 Einen ähnlichen Vorschlag für die interne Ausdifferenzierung der Utopie findet sich bei Darko Suvin: „Utopia may be divided into the polar opposites of: EUTOPIA, [...] having the sociopolitical institutions, norms, and relationships between people organized according to a $r a-$ dically more perfect principle than in the author's community; and DYSTOPIA (cacotopia), organized according to a radically less perfect principle. The radical difference in perfection is in both cases judged from the point of view and within the value-system of a discontented social class or congeries of classes, as refracted through the writer.“ (Darko Suvin: A Tractate on Dystopia 2001. In: Ders.: Defined by a Hollow. Essays on Utopia, Science Fiction and Political Epistemology. Bern 2010, S. 381-412, hier S. 382).

1035 Layh: Finstere neue Welten, S. 25.

1036 Besonders in der Forschung zu Gegenwartsliteratur und -film ist der Terminus ,Dystopie‘ gut eingeführt. Gelegentlich anzutreffende Alternativbegriffe wie etwa Anti-Utopie, negative Utopie, Kakotopie, Mätopie oder Warnutopie bringen analytisch kaum einen Mehrwert und werden de facto meist synonym mit dem Begriff Dystopie gebraucht, sodass sich die Entscheidung für nur einen dieser Begriffe empfiehlt. Zur Terminologiediskussion siehe Stephan Meyer: Die anti-utopische Tradition. Eine ideen- und problemgeschichtliche Darstellung. Frankfurt a. M. 2001, S. 17-33; Artur Blaim: Dystopia, Anti-utopia \& Co. Another Modest Proposal. In: Ders.: Utopian Visions and Revisions Or the Uses of Ideal Worlds. Frankfurt a. M. 2017, S. 11-21. Produktiv wird speziell der Terminus der ,Anti-Utopie‘ dann, wenn man dessen gattungsreflexive Dimension hervorhebt, also anti-utopische Texte nicht primär mit Blick auf das in ihnen entworfene Gesellschaftsmodell, sondern als „Phänomen der literarisch verkleideten Utopiekritik“ diskutiert (Meyer: Die anti-utopische Tradition, S. 31). Suvin differenziert in diesem Sinne zwischen „anti-utopia“ und „'simple’ dystopia“ (Suvin: A Tractate on Dystopia 2001, S. 385). Für eine Unterscheidung von Dystopie und Anti-Utopie plädiert auch Layh: Finstere neue Welten, S. 110-115. Zeißler hingegen betont, dass die genrekritische Funktion der Dystopie/Anti-Utopie in der Gegenwart an Relevanz verloren habe: „Mit dem Schwinden der Bedeutung der klassischen, geschlossenen Utopie aus dem öffentlichen Bewußtsein erübrigt sich eine der Aufgaben der traditionellen Dystopie fast gänzlich, nämlich die der Parodie auf das utopische Denken.“ (Zeißler: Dunkle Welten, S. 223). 
Die systematische Einbeziehung von utopisch/dystopischen Texten in ein mögliches Untersuchungskorpus der Kontrafaktik bildet innerhalb der literaturwissenschaftlichen Forschung eine Neuerung, welche der Begründung bedarf. Während sich, wie im Kapitel zum Zusammenhang von Kontrafaktik und Dokumentarismus ausgeführt wurde, die kontrafaktischen Potenziale dokumentarischen Erzählens noch relativ mühelos an diejenigen des historischen Erzählens anschließen lassen (in beiden Fällen beziehen sich die zu variierenden Fakten offenkundig auf die Vergangenheit), mag die Subsumption utopisch/ dystopischer Zukunftserzählungen unter das Untersuchungsraster der Kontrafaktik zunächst befremdlich wirken. Dass und weshalb eine solche Subsumption gleichwohl sinnvoll, ja aus einer bestimmten fiktionstheoretischen Perspektive betrachtet sogar unausweichlich ist, soll im Folgenden dargelegt werden.

Auf eine mögliche Verbindung zwischen kontrafaktischem und utopisch/dystopischem Erzählen ist in der Forschung immer wieder hingewiesen worden. So schreibt etwa Wilhelm Voßkamp für das Genre der Zeitutopien von einer „(kontrafaktischen) Antizipation des Zukünftigen“. ${ }^{1037}$ Johannes Birgfeld und Claude D. Conter bemerken zum Alternativgeschichtsroman: „Der Übergang des Genres zur Science-Fiction ist fließend, die Nähe zu Utopie und Dystopie groß.“1038 Und Frank Finlay konstatiert: „Counter-factual history, of course, lends itself to dystopian and utopian visions of the future, which themselves can be used to provide a critical rear-view mirror on the culture, society and politics of the present or past.“1039 Trotz der offenbar weitverbreiteten Intuition, dass kontrafaktisches und utopisch/dystopisches Erzählen eine enge Verbindung miteinander aufweisen, wurde diese Verbindung bisher nicht systematisch entwickelt. Birte Christ immerhin weist auf das entsprechende Forschungsdesiderat sowie auf die mögliche Produktivität einer Betrachtung utopisch/dystopischer Texte unter dem Analyseraster kontrafaktischen Denkens hin:

Surprisingly [...] the classical literary utopia in the tradition of Thomas More and, more widely framed, speculative fictions with a utopian impetus have so far not been explored from the perspective of counterfactuality. [...] Here is an entire literary genre which may be read afresh from the perspective of the studies of counterfactuality [...]. ${ }^{1040}$

1037 Wilhelm Voßkamp: Einleitung. In: Ders. (Hg.): Utopieforschung. Interdisziplinäre Studien zur neuzeitlichen Utopie. 3 Bde. Stuttgart 1982. Bd. 1, S. 1-10, hier S. 6.

1038 Birgfeld / Conter: Morgenröte des Posthumanismus, S. 258.

1039 Finlay: 'Surface is an illusion but so is depth', S. 221.

1040 Birte Christ: "If I Were a Man": Functions of the Counterfactual in Feminist Fiction. In: Dorothee Birke / Michael Butter / Tilmann Köppe (Hg.): Counterfactual Thinking - Counterfactual Writing. Berlin 2011, S. 190-211, hier S. 220 f. 
Über die Gründe für dieses Versäumnis der Forschung kann letztlich nur spekuliert werden; einige begründete Vermutungen lassen sich aber immerhin anstellen. Wie im Rahmen der Forschungsdiskussion bereits ausgeführt wurde, dürfte der literaturwissenschaftliche Fokus auf die Kontrafaktik im historischen Roman den Blick für Verfahren signifikanter Faktenvariation in anderen Genres weitgehend verstellt haben. Betroffen sind hiervon etwa Realitätsvariationen in der Autofiktion, der Satire, dem Schlüsselroman und eben auch der Utopie/Dystopie. ${ }^{1041}$

Über diese genreübergreifenden Einschränkungen hinaus dürfte im speziellen Fall der Utopie/Dystopie vor allem die Zukünftigkeit der Erzählwelten eine Betrachtung der jeweiligen fiktionalen Werke unter dem Vorzeichen der Kontrafaktik behindert haben. Diese Zukünftigkeit der Diegese gibt zwei Einwänden gegen die Betrachtung utopisch/dystopischer Texte als Werke der Kontrafaktik Raum, denen im Folgenden begegnet werden soll: Erstens könnte man vorbringen, dass ,Fakten' der Zukunft ja gar nicht variiert werden könnten, da die Zukunft noch nicht eingetreten sei und entsprechend auch kein variationsfähiges Faktenmaterial bereitstelle. Und zweitens könnte man die Meinung vertreten, dass Utopien/ Dystopien ja gar keine ,Variationen“ realweltlichen Faktenmaterials, sondern vielmehr mögliche Folgenabschätzungen realer Entwicklungen liefern würden.

Was die vermeintlichen Zukunftsfakten angeht, so ist zunächst zu konzedieren, dass moderne Utopien/Dystopien tatsächlich oft futurische Räume entwerfen. Nachdem in der vormodernen Literatur räumliche Utopien, etwa Erzählungen von weit entfernten Inselstaaten, dominiert hatten, setzt um 1800 eine, wie Reinhart Koselleck schreibt, „Verzeitlichung der Utopie“1042 ein, sodass die Erzählwelten der meisten modernen Utopien/Dystopien in der Zukunft angesiedelt sind. ${ }^{1043}$ (Allerdings wird mit Leif Randts Schimmernder Dunst über CobyCounty im Folgenden ein Roman diskutiert, der auf gegenwartsuntypische Weise eher eine „Raumutopie“ als eine „Zeitutopie“ entwirft. ${ }^{1044}$ ) Für eine fiktions- und interpretationstheoretisch präzise Beschreibung von Zukunftsgenres wie Utopie und Dystopie, aber

1041 Freilich sind auch Hybridbildungen zwischen verschiedenen Genres möglich. So erfüllen etwa Schlüsselromane mitunter satirische Funktionen. Auch steht die Dystopie in enger Verbindung zur Satire. Vgl. Suvin: A Tractate on Dystopia 2001, S. 386f. Zum Zusammenhang von Autofiktion und Utopie siehe Yvonne Delhey / Rolf Parr / Kerstin Wilhelms (Hg.): Autofiktion als Utopie // Autofiction as Utopia. Paderborn 2019.

1042 Vgl. Reinhard Koselleck: Die Verzeitlichung der Utopie. In: Wilhelm Voßkamp (Hg.): Utopieforschung. Interdisziplinäre Studien zur neuzeitlichen Utopie. 3 Bde. Stuttgart 1982. Bd. 3, S. 1-14.

1043 Vgl. Thomas Schölderle: Geschichte der Utopie. 2., überarbeitete und aktualisierte Aufl. Weimar / Köln / Wien 2017, S. 11.

1044 Zur geschichtlichen Unterscheidung von Raum- und Zeitutopien siehe Wilhelm Voßkamp: Narrative Inszenierung von Bild und Gegenbild. Zur Poetik literarischer Utopien. In: 
auch für die Science-Fiction und für apokalyptische Erzählungen ist es nun allerdings von großer Wichtigkeit, zwischen der Ebene der fiktionalen Welt und der Interpretationsebene zu unterscheiden: Nur weil die Erzählwelten von Utopie/ Dystopie in der Zukunft angesiedelt sind, folgt daraus noch nicht, dass die Fakten, auf die sich diese Erzählwelten beziehen, selbst auch notwendigerweise ,aus der Zukunft stammen“. Auf der Ebene der Interpretation verweisen Utopien/ Dystopien in aller Regel nicht oder nicht primär auf eine als möglich gedachte Zukunft, sondern vielmehr auf Konkreta der Gegenwart; der Weltentwurf utopisch-dystopischer Texte deutet also nicht in gegenwartsindifferenter Weise in die Zukunft, sondern liefert anhand eines fiktiven Zukunftssettings eher einen kritischen Kommentar zu aktuellen gesellschaftlichen oder technischen Entwicklungen. So betont Elena Zeißler, dass die Gesellschaftsentwürfe jüngerer Dystopien „als lediglich verzerrte oder allegorische Bilder der Gegenwart und nicht als Antizipation der Zukunft zu verstehen“ seien. ${ }^{1045}$ Das politische Potenzial utopisch/dystopischer Texte leitet sich für gewöhnlich gerade aus der Verbindung zwischen der futurisch-fiktionalen Diegese und ihrer realweltlich-gegenwärtigen Bezugsebene ab, die es im Rahmen der Interpretation zu erschließen gilt.

Die futurische Verortung von utopisch/dystopischen Erzählwelten ist also meist nicht so sehr Ausdruck eines gesteigerten Interesses an möglichen Zukunftsentwicklungen, sondern dient eher der Eröffnung eines Freiraums für die fiktional ungebundene Kommentierung gegenwärtiger gesellschaftlicher oder politischer Entwicklungen. Georg Ruppelt bemerkt in diesem Sinne mit Blick auf ein Korpus von rund 130 Zukunftserzählungen:

Eines ist den Texten bekannter wie unbekannter Autoren gemeinsam: sie geben eindrucksvoll Auskunft über den Zeitgeist; natürlich nicht über den der in ihnen vorgestellten mehr oder weniger fernen Zukunft, sondern über den Zustand jener Gesellschaft, deren Glied der jeweilige Autor ist.

Denn durch die Verlagerung ihrer Geschichten in die Zukunft scheinen viele Autoren sich selbst die Erlaubnis zu gewähren, gewisse Hemmungen abzulegen und manches durch Überzeichnung besonders deutlich hervorzuheben. Infolgedessen ist es möglich, daß sich in diesen Zukunftsgeschichten die Ängste und Hoffnungen des Autors und seiner Zeit wesentlich elementarer als in anderen Textsorten offenbaren. ${ }^{1046}$

Es lässt sich festhalten: In Utopien/Dystopien werden Gegenwartstendenzen in die Zukunft projiziert und innerhalb der fiktionalen Welten variiert, um dadurch Ent-

Árpád Bernáth / Endre Hárs / Peter Plener (Hg.): Vom Zweck des Systems. Beiträge zur Geschichte literarischer Utopien. Tübingen 2006, S. 215-226, hier S. 217-223.

1045 Zeißler: Dunkle Welten, S. 224.

1046 Georg Ruppelt: Zukunft von gestern. In: Heyne Science Fiction Magazin 11 (1984), S. 181-232, hier S. 183. 
wicklungen der Gegenwart kritisch zu kommentieren. Diese Anbindung an die Gegenwart eröffnet dabei zugleich vielfältige Möglichkeiten für den Einsatz der Kontrafaktik. Wenn etwa George Orwell in seinem Roman 1984 von den Geschichtsklitterungen im Ministry of Truth erzählt, so wird damit nicht auf eine Zukunftsentwicklung hingewiesen; vielmehr werden hier die realen Geschichtsfälschungen des Stalinismus kontrafaktisch variiert und damit kritisch kommentiert (darüber hinaus handelt es sich beim Ministry of Truth um ein besonders prominentes Beispiel eines metafaktischen Elements, wird über die Beschreibung der Arbeitsweise des Ministeriums doch das Thema der Geschichtsdeviation in die erzählte Welt des dystopisch-kontrafaktischen Texts hineingeholt ${ }^{1047}$ ).

Der zweite Einwand gegen eine Verbindung von Kontrafaktik und utopisch/dystopischem Erzählen betrifft den vermeintlich prognostischen Charakter des Genres. Nähme man nämlich an, Utopien/Dystopien würden mögliche oder wahrscheinliche Zukunftsentwicklungen der realen Gesellschaft antizipieren, so müsste die Option kontrafaktischer Interpretationen in der Tat ausfallen. Bei derartigen Prognosen würde es sich gerade nicht um kontrafaktische - ihrem Gehalt nach also offenkundig fiktive - Aussagen handeln, sondern vielmehr um Folgenabschätzungen realer Entwicklungen, gewissermaßen also um eine Form der Zukunftsrealistik. Ein einfaches Beispiel sei angeführt: Die in Bezug auf die reale Welt geäußerte Prognose „Morgen wird es regnen“ ist nicht oder doch nur in einem sehr reduzierten Sinne als kontrafaktisch anzusehen. Zwar wird hier auf einen fiktiven Sachverhalt, nämlich die künftige Wetterlage, Bezug genommen; ein realweltliches Vergleichselement jedoch respektive eine konkrete Doppelreferenz von Fakt und Kontrafakt, wie sie für die Kontrafaktik als Erzählverfahren konstitutiv ist, fehlt. Darüber hinaus handelt es sich bei der realen Zukunftsprognose „Morgen wird es regnen“ um eine faktuale Aussage, eine Aussage also, die sich auf die reale Welt bezieht und mithin eine etwaige prognostische Plausibilität auch nur anhand von Daten der realen Welt gewinnen kann, etwa indem ein Wetterdienst auf der Basis von Messwerten zur aktuellen Wetterlage und unter Zuhilfenahme meteorologischer Kalkulationsmodelle Tendenzaussagen über die zukünftige Wetterentwicklung trifft.

Bei literarischen Utopien/Dystopien hingegen handelt es sich um fiktionale Texte, die nicht notwendigerweise an die epistemischen Einschränkungen einer faktualen Futurologie gebunden sind. Ebenso wie im Bereich des kontrafaktischen Denkens und Erzählens eine Trennung zwischen dem Feld der Faktualität (Kontrafaktizität) und dem Feld der Fiktionalität (Kontrafaktik) sinnvoll

1047 Siehe Kapitel 5.4. Metafaktizität. 
ist, so sollte auch beim futurischen Denken unterschieden werden zwischen Texten und Medien mit einem faktualen, streng szientifisch-prognostischen Geltungsanspruch einerseits (Futurologie) und fiktionalen, künstlerischen Texten und Medien andererseits (literarische Dystopien, Utopien und ScienceFiction-Texte). ${ }^{1048}$ Für die Interpretation literarischer Utopien/Dystopien ist die Frage ihrer futurologischen Plausibilität meist von untergeordneter Bedeutung. Dies lässt sich schon daran erkennen, dass Utopien/Dystopien ihr politisches Potenzial auch dann nicht einbüßen, wenn sie über die zeitliche Verortung ihrer Zukunftsdiegese hinausaltern: Orwells 1984 etwa wurde im realen Jahr 1984 als Dystopie keineswegs bedeutungslos. Tatsächlich erweisen sich viele utopisch/dystopische Texte als auffallend indifferent gegenüber der realweltlichen Plausibilität oder auch nur Wahrscheinlichkeit ihrer Zukunftsvisionen. Würde man strenge Kriterien faktualer Plausibilität und argumentativer Stringenz an den Kanon utopisch/dystopischer Texte anlegen, so würde sich ein ähnlicher Effekt einstellen wie bei dem Versuch, die Einschränkungen des faktual-historiografischen Diskurses auf den Kanon alternativgeschichtlicher literarischer Werke anzuwenden: Die Mehrzahl der Texte, inklusive der meisten Klassiker des jeweiligen Genres, müsste aus dem Kanon ausscheiden.

Insbesondere für die politische Evaluation utopisch/dystopischer Texte dürfte weniger ihre prognostische Dimension als ihr (kontrafaktischer) Gegenwartsbezug ausschlaggebend sein. Wenn etwa Michel Houellebecq im Roman Unterwerfung eine Islamisierung Frankreichs im Jahr 2022 imaginiert, so spielt es für die Interpretation und politische Evaluation des Romans kaum eine Rolle, ob eine solche Entwicklung im Jahre 2022 tatsächlich eintreten wird oder auch nur eintreten könnte; relevant sind vielmehr jene gesellschaftlichen Zustände und argumentativen Zusammenhänge, aus denen heraus der Roman entstanden ist und in die er hineinwirkt. Ob es sich also bei Unterwerfung etwa um einen islamophoben Text handelt - was an dieser Stelle nicht diskutiert werden soll -, hängt nicht davon ab, ob Frankreich im Jahre 2022 tatsächlich eine islamische Regierung bekommen wird (so wenig wie das politische Potenzial von Orwells 1984 davon abhing, ob Big Brother die englische Bevölkerung im Jahre 1984 tatsächlich überwachen würde); für eine politische Evaluation von Houellebecqs Roman entscheidend ist vielmehr die literarische Variation aktueller gesellschaftlicher Verhältnisse und Positionen der öffentlichen Meinungen, also etwa: die rechtspopulistische Angst vor einer Islamisierung

1048 Siehe zu unterschiedlichen Ansätzen der Futurologie Benjamin Bühler / Stefan Willer (Hg.): Futurologien. Ordnungen des Zukunftswissens. Paderborn 2016. Die Beiträge dieses Bandes sind allerdings nicht auf faktuale Medien beschränkt. 
Frankreichs, die Verbindung von orthodoxer Religiosität und Geburtenraten, die wirtschaftliche, moralische und kulturelle ,Dekadenz des Westens', die sozialen, bildungsbezogenen und sexuellen Möglichkeiten der verschiedenen Geschlechter und nicht zuletzt der politische Opportunismus einer wirtschaftlich und libidinös frustrierten Mittelschicht. ${ }^{1049}$ Sofern es sich bei diesen Themen um ,Fakten“ der Gegenwart handelt, kann ihre künstlerische Abwandlung innerhalb der futurischen Diegese von Houellebecqs Roman als kontrafaktische Variation gedeutet werden - und zwar ganz unabhängig von ihrer futurologischen Plausibilität (die bei Unterwerfung im Übrigen eher gering sein dürfte ${ }^{1050}$ ).

Bei den in Forschung und Feuilleton nicht selten zu beobachtenden Versuchen, Utopien/Dystopien primär an Kriterien ihrer prognostischen Plausibilität oder gar faktischen Richtigkeit zu messen, handelt es sich wiederum um einen Fall epistemischer Übergeneralisierung: Bewertungskriterien aus dem faktualen Bereich werden an literarische Texte herangetragen und damit die besonderen Lizenzen und Potenziale fiktionalen Erzählens ignoriert. ${ }^{1051}$ Derartige epistemische Übergeneralisierungen drohen unter anderem da, wo von Dystopien als ,Warnutopien ${ }^{\text {1052 }}$ gesprochen wird; schließlich lassen sich ,Warnungen' strenggenommen

1049 Vgl. Navratil: Jenseits des politischen Realismus, S. 372.

1050 In einem polemischen Artikel weist der Zeit-Herausgeber Josef Joffe auf die sachlichen Unstimmigkeiten von Houellebecqs Roman hin. Vgl. Josef Joffe: Mon dieu, Michel. Houellebecq fantasiert über die Islamisierung Frankreichs. In: Die Zeit, 15.01.2015.

1051 Tatsächlich scheint das utopische Denken, ähnlich wie das kontrafaktische Denken in der Geschichte, einen natürlichen Zug hin zum fiktionalen Erzählen aufzuweisen oder zumindest freie Erfindungslizenzen, wie sie für fiktionale Texte charakteristisch sind, für sich zu behaupten. Zurecht betont Jörn Rüsen, dass das utopische Denken seine Produktivität wesentlich aus der Überschreitung der Grenzen des real Machbaren bezieht: „Soll nur dasjenige kulturell handlungsmotivierend wirken, was als machbar gilt? Dann wären Utopien in der Tat überflüssig geworden. [...] Mit den Potentialen des Utopischen geht es um die inspirierende und phantasievolle Kraft des Überschreitens von hemmenden Grenzen, ja generell um die geistige Kraft des Überschwenglichen in den kulturellen Deutungen und Sinnbestimmungen des menschlichen Lebens.“ (Jörn Rüsen: Einleitung: Utopie neu denken. Plädoyer für eine Kultur der Inspiration. In: Ders. / Michael Fehr / Annelie Ramsbrock (Hg.): Die Unruhe der Kultur. Potentiale des Utopischen. Weileswist 2004, S. 9-23, hier S. 14).

1052 Vgl. Layh: Finstere neue Welten, S. 159; Voßkamp: Utopie, S. 744; Hiltrud Gnüg: Warnutopie und Idylle in den Fünfziger Jahren. Am Beispiel Arno Schmidts. In: Dies. (Hg.): Literarische Utopie-Entwürfe. Frankfurt a. M. 1982, S. 277-290. Hiltrud Gnüg weist allerdings zurecht darauf hin, dass sich die Idee einer „Warnutopie“ auch in Beziehung zur Gegenwart setzen lässt: „[Es] manifestiert sich das utopische Denken nicht allein in einem Vorgriff auf eine bessere Zukunft, sondern auch im antizipatorischen Kassandra-Blick auf die negativen Möglichkeiten, die in der Gegenwart angelegt sind. Auch hier erweist sich die utopische Phantasie, die den Ist-Zustand transzendiert, als Imaginationskraft, die - mit dem Faktisch-Bestehenden vermittelt - dieses 
nur auf faktisch plausible Zukunftsentwicklungen beziehen. ${ }^{1053}$ Als nicht weniger problematisch erweist sich die Kategorie der ,Möglichkeit‘ oder des ,Möglichkeitsdenkens', auf die bei der Diskussion rund um Utopien/Dystopien ähnlich häufig zurückgegriffen wird wie bei der Diskussion des kontrafaktischen Erzählens. ${ }^{1054}$ Es wurde bereits ausgeführt, dass das ursprünglich modallogische Konzept der möglichen Welten (possible worlds) sich nur bedingt auf fiktionale Texte übertragen lässt. ${ }^{1055}$ Aber auch unabhängig von einem derartig spezifischen Gebrauch ist der Begriff der ,Möglichkeit‘ für die Bewertung fiktionaler Texte problematisch, verleitet er doch dazu, Bewertungskriterien faktualer Diskurse (Plausibilität, Wahrscheinlichkeit, Widerspruchsfreiheit, empirische Überprüfbarkeit etc.) auch an fiktionale Texte heranzutragen, an Texte also, die qua Fiktionalität eigentlich von derartigen Bewertungskriterien unabhängig sein sollten. ${ }^{1056}$ Freilich lässt sich auch für fiktionale Zukunftsdiegesen versuchsweise

doch zugleich kritisiert, es von der Vorstellung einer besseren Lebensmöglichkeit her radikal in Frage stellt.“ (Hiltrud Gnüg: Einleitung. In: Dies. (Hg.): Literarische Utopie-Entwürfe. Frankfurt a. M. 1982, S. 9-14, hier S. 13f.) Damit bindet auch Gnüg die Idee des Utopischen gleichsam vorbegrifflich an die Kontrafaktik.

1053 Zeißler macht darauf aufmerksam, dass sich die „Rolle der Zukunft“ in neueren Dystopien stark verändert hat, sodass „die Warnfunktion, die bei Huxley und Orwell noch eine zentrale Rolle spielte, nicht mehr so stark ausgeprägt ist. Konnte der Leser der klassischen AntiUtopien deren Entwürfe noch für theoretisch realisierbar halten (weswegen sie manchmal auch als Prophezeiungen gedeutet wurden), so dient jetzt die Verlagerung der Handlung in die Zukunft nur dem Entwurf eines parallelen Raumes, der die Wirklichkeit des Lesers widerspiegeln soll.“ (Zeißler: Dunkle Welten, S. 223).

1054 Siehe beispielsweise Wilhelm Voßkamp / Günter Blamberger / Martin Roussel (Hg.): Möglichkeitsdenken. Utopie und Dystopie in der Gegenwart. München 2013; Esselborn: Vorwort, S. 8-10; Inga Ketels: Der Einzug des Politischen in die Gegenwartsliteratur. Imaginierte Alternativen als Neuverhandlung von Möglichkeitsräumen bei Christian Kracht, Juli Zeh und Dorothee Elmiger. In: Gillian Pye / Sabina Strümper-Krobb (Hg.): Germanistik in Ireland 9. Special Issue: Imagining Alternatives: Utopias - Dystopias - Heterotopias. Konstanz 2014, S. 105-120; Doležel: Possible Worlds of Fiction and History.

1055 Siehe Kapitel 3.1. Positionen der Forschung.

1056 Eine solche Entdifferenzierung von faktualen und fiktionalen Deutungsansprüchen lässt sich etwa bei Eva Horn (Zukunft als Katastrophe) beobachten, wenn sie schreibt: „,Fiktionalität‘ (sei es von wissenschaftlichen Szenarien, sei es von Romanen oder Filmen) in Hinblick auf eine Beleuchtung künftiger Welten bedeutet, dass sich Zukunft gleichsam aufspaltet in einen möglichen Verlauf, der erwartet, vorausgesagt, möglicherweise verhindert oder herbeigeführt werden kann - und einen anderen, ebenso möglichen, der nicht erwartet, nicht gewusst, nicht verhindert werden kann.“ (ebd., S. 303) Problematischer als die offenkundige Verwechslung von Fiktivität und Fiktionalität - ,Fiktionalität wissenschaftlicher Szenarien“ ist strenggenommen ein Oxymoron - erweist sich hier die Bindung von Zukunftsimaginationen an die Kategorie des Möglichen, durch die stark spekulative oder fantastische Zukunftsszena- 
die Frage stellen, ob die in ihnen entworfenen Szenarien tatsächlich eintreten könnten; man sollte sich in einem solchen Fall aber stets vor Augen halten, dass hier Bewertungskriterien aus dem Bereich der Faktualität an einen fiktionalen Text herangetragen und mithin bestimmte pragmatische, gesellschaftlich institutionalisierte Geltungsansprüche - diejenigen der Wissenschaft und der Kunst nämlich miteinander vermischt werden. ${ }^{1057}$

Generell ist das kontrafaktische Potenzial futurischer Erzählwelten nicht an die ,Möglichkeit‘ ihrer faktischen Realisierung gebunden. ${ }^{1058}$ Tatsächlich sind bei fiktionalen Zukunftstexten alle vier logischen Kombinationsmöglichkeiten zwischen den Kategorien der (Un-)Möglichkeit und den Kategorien der (nicht vorhandenen) Kontrafaktik vorstellbar, wie die folgende Liste fiktiver Beispiele demonstriert:

- Möglich und nicht kontrafaktisch: In einem Roman löscht ein Meteor im Jahr 2050 völlig unverhofft alles Leben auf der Erde aus. Hier läge tendenziell kein kontrafaktischer Text vor, da das Ereignis des Meteoriteneinschlags nicht produktiv kontrastierend auf ein Faktum der Gegenwart verweist. Bezeichnenderweise fällt es nicht leicht, Beispiele für diese Textgruppe zu finden, wohl deshalb, weil Zukunftsvisionen, die sich interpretatorisch nicht in irgendeiner Weise auf die Gegenwart beziehen lassen, zugleich aber auch auf fantastische Elemente verzichten, nur von geringem Interesse für die Le-

rien, wie Horn sie in ihrem Buch durchaus diskutiert, strenggenommen aus dem Untersuchungskorpus ausgeschlossen werden müssten.

1057 Man könnte hier eine Unterscheidung zwischen eher futurologisch-epistemisch orientierten und eher fiktional-künstlerisch orientierten Lesarten fiktionaler Utopien/Dystopien einführen, wobei fiktional-kontrafaktische Lesarten der zweiten Gruppe zuzuschlagen wären. Während futurologische Lesarten der Utopie/Dystopie die plausible Verbindung zwischen Gegenwart und Zukunft betonen und danach fragen, ob bestimmte Entwicklungen in der Zukunft tatsächlich eintreten könnten - also fiktionale Texte gewissermaßen als pseudo-faktuale Texte behandeln -, heben kontrafaktische Lesarten insbesondere der Dystopie eher ihre indirekten Gegenwartsreferenzen, ihren satirischen Charakter und ihre Funktion als Kommentar zum aktuellen Zeitgeist hervor. Es handelt sich hier allerdings nicht um zwei einander ausschließende Deutungsoptionen, sondern eher um unterschiedliche interpretatorische Schwerpunktsetzungen: Mitunter lassen sich sowohl futurologische als auch kontrafaktische Lesarten an ein und denselben Text herantragen. Zumindest in der Literaturwissenschaft verbreiteter und auch für eine politische Lesart der jeweiligen Texte relevanter dürfte jedoch die Option kontrafaktischer Interpretationen sein.

1058 Prinzipiell können kontrafaktische Elemente zugleich auch fantastisch sein, müssen es aber nicht. Siehe Kapitel 5.1. Realistik, Fantastik, Kontrafaktik, Faktik. So finden sich in Werken der historischen Kontrafaktik mitunter fantastische - also realweltlich unmögliche - Elemente, die zugleich auch als kontrafaktische Elemente fungieren, etwa die fantastische Figur Dr. Manhattan im Comic Watchmen, welcher den Amerikanern durch seine übernatürlichen Kräfte zum Sieg im Vietnam-Krieg verhilft. 
serschaft fiktionaler Texte sind (während vergleichbare Szenarien für die $\mathrm{Zu}$ kunftseinschätzungen einer faktualen Futurologie durchaus relevant sein können). ${ }^{1059}$

- Unmöglich und nicht kontrafaktisch: Aufgrund einer technischen Innovation im Jahr 2050 beginnt die Zeit mit einem Mal rückwärts zu laufen. Ein solches Ereignis ist unter Wahrung aktuell geltender physikalischer Realitätsannahmen nicht möglich. Es wäre aber auch nicht eigentlich kontrafaktisch, da hier kein realweltliches Faktum (abgesehen von der sehr allgemeinen Annahme eines monodirektionalen Zeitverlaufs) variiert wird. In einem solchen Fall hätte man es eher mit einem Werk der Fantastik zu tun. Ein Großteil der (Zukunfts-)Erzählungen über eine Invasion der Erde durch Außerirdische etwa H. G. Wells' Science-Fiction-Klassiker The War of the Worlds (1897) oder Roland Emerichs Katastrophenblockbuster Independence Day (1996) - lassen sich dieser Gruppe zuschlagen, da sie strenggenommen weder als möglich noch als kontrafaktisch zu klassifizieren sind. ${ }^{1060}$

- Möglich und kontrafaktisch: Im Jahr 2050 leben alle Menschen unter den Bedingungen einer digitalen Überwachungsdiktatur. Eine solche Entwicklung ist insofern möglich, als sie sich vom heutigen Standpunkt zumindest nicht ausschließen lässt. Kontrafaktisch wäre ein solcher Weltentwurf darüber hinaus in dem Maße, in dem er sich interpretatorisch auf aktuelle technische oder politische Entwicklungen oder auf Einzelfakten der Gegenwart (oder auch der Vergangenheit) beziehen lässt. Die meisten fiktionalen Dystopien lassen sich diesem Typ zuschlagen. Mit Juli Zehs Roman Corpus Delicti wird im Folgenden ein Beispiel genau dieses Typs diskutiert.

1059 Katastrophenblockbuster wie Michael Bays Armageddon (1998) oder Mimi Leders Deep Impact (1998) erzählen gerade nicht von einem alles vernichtenden Meteoriteneinschlag auf der Erde, wie er ja durchaus im Vorstellungsbereich einer faktualen Futurologie liegt, sondern von der technischen Abwendung eines solchen. Die potenzielle Katastrophe erfüllt hier vor allem eine motivationale Alibifunktion, indem sie die Verhandlung von Themen wie Heldentum, familiäre Konflikte und Patriotismus ermöglicht.

1060 Häufig plausibilisieren Science-Fiction-Erzählungen die Entstehung ihrer de facto fantastischen Diegese über eine pseudo-wissenschaftliche Erklärung. So bemerkt Hans-Edwin Friedrich zur Gattungsdefinition der Science-Fiction: „Als Kern der Definition lässt sich die Beschaffenheit der jeweils entworfenen Fiktion bestimmen: Sie ist auf eine besondere Weise fantastisch; gegenüber realistisch konzipierten Fiktionen ist sie durch das Vorhandensein mindestens eines abweichenden Novums gekennzeichnet. Dieses Novum beruht auf einer rationalen Extrapolation und muss naturwissenschaftlich begründet sein, wobei diese Absicherung in einer strengen Variante logisch aus dem zur Zeit der Niederschrift des Textes gängigen naturwissenschaftlichen Kenntnisstand abzuleiten ist; in einer freieren Variante genügt eine wissenschaftliche Scheinerklärung.“ (Friedrich: Science-Fiction, S. 673f.). 
- Unmöglich und kontrafaktisch: Im Jahre 2050 verbünden sich sämtliche Tiere, um fortan die menschliche Rasse unter grausamen Bedingungen als Nutzwesen zu halten (eine ähnliche Zukunftsvision entwirft Dietmar Dath in seinem Roman Die Abschaffung der Arten (2008)). Eine derartige Entwicklung ist mit den Annahmen der heutigen Zoologie unvereinbar und entsprechend als fantastisch einzustufen. Dies schließt jedoch nicht aus, dass hier zugleich auf kontrafaktische Referenzstrukturen zurückgegriffen wird, etwa indem die fiktive Handlung als kritischer Kommentar zu einem aktuellen Tierhaltungsskandal interpretiert würde. Dieses Beispiel lässt nicht zuletzt erkennen, dass die zeitliche Verortung der Handlung letztlich von untergeordneter Bedeutung für die Identifikation kontrafaktischer Elemente ist: $\mathrm{Ob}$ der genannte Aufstand der Tiere, welcher kontrafaktisch auf einen Skandal der Gegenwart Bezug nimmt, als Science-Fiction in die Zukunft oder als Alternativgeschichte in die Vergangenheit projiziert wird, hat keinen Einfluss auf den kontrafaktischen Status dieses Elements.

Im Rahmen eines Referenzmodells der Kontrafaktik ist es also - zumindest für die Bestimmung der kontrafaktischen Elemente - vorderhand unerheblich, ob fiktionale Zukunftswelten in ihrer Gesamtheit mögliche oder unmögliche Zukunftsverläufe aufzeigen (was nicht heißt, dass die Unterscheidung zwischen Möglichkeit und Unmöglichkeit nicht mitunter für die Interpretation der jeweiligen Texte von Interesse sein kann). Ausschlaggebend für die Identifikation einer kontrafaktischen Referenzstruktur ist lediglich der interpretatorisch relevante, indirekte Bezug auf Realitätsannahmen, welche in der Gegenwart der Leserschaft als Fakten akzeptiert sind.

Für eine Betrachtung utopisch/dystopischer Texte als Werke der Kontrafaktik sehr viel wichtiger als die Frage nach der (Un-)Möglichkeit von Erzählwelten ist die Frage, welches Faktenmaterial hier überhaupt als Basis einer kontrafaktischen Faktenvariation herangezogen werden kann; wann also lässt sich beim realweltlichen Bezugsmaterial der fiktionalen Welten von Utopien/Dystopien sinnvollerweise von ,Fakten“ sprechen? Es wurde bereits ausgeführt, dass die historische Kontrafaktik in aller Regel auf hochgradig konventionalisiertes Faktenmaterial zurückgreift, auf Aussagen über die Vergangenheit also, deren Wahrheitswert erstens breit akzeptiert ist und die zweitens über einen hohen Bekanntheitsgrad verfügen, sodass zuverlässig davon ausgegangen werden kann, dass die Variation dieser Fakten innerhalb einer fiktionalen Welt als solche erkannt und in der Folge interpretatorisch produktiv gemacht wird. ${ }^{1061}$ Nun bietet

1061 Siehe Kapitel 3.2.3. Kontrafaktisches Erzählen als Genrevariante historischen Erzählens. 
sich prinzipiell auch für Utopien/Dystopien die Möglichkeit, auf historisches Faktenmaterial zurückzugreifen. In Timur Vermes' Roman Er ist wieder da (2012) oder in Timo Vuorensolas Science-Fiction-Satire Iron Sky: The Coming Race (2019) treten etwa wiederauferstandene Versionen Adolf Hitlers auf. Allerdings wird die Einführung dieser Figuren in den genannten Werken nicht dazu genutzt, die Geschichte des Dritten Reiches kontrafaktisch zu variieren; stattdessen werden sie lediglich als isolierte kontrafaktische Elemente in eine neue (futurische) Erzählwelt verpflanzt. Die genannten (dystopischen) Werke beinhalten also kontrafaktische Elemente mit historischem Bezugsmaterial, ohne deshalb dem Genrebereich der Alternate History zugeordnet werden zu müssen.

Für gewöhnlich beziehen sich Utopien/Dystopien jedoch nicht auf historisches Faktenmaterial, sondern auf aktuelle gesellschaftliche oder politische Entwicklungen, Tendenzen und Diskurse, gelegentlich auch auf Einzelereignisse der Gegenwart/jüngsten Vergangenheit oder auf Personen des öffentlichen Lebens. So verweist die Darstellung eines hyperbolischen Konsumismus und Kapitalismus in Aldous Huxleys Roman Brave New World zweifelsohne auf reale Wirtschaftstendenzen zur Entstehungszeit des Romans.

Derartige Bezugnahmen utopisch/dystopischer Texte auf gesellschaftliche Trends und Diskurse der Gegenwart werfen für kontrafaktische Interpretationen allerdings ein Problem auf: Verglichen nämlich mit konkreten historischen Informationen - etwa dem Todesjahr bestimmter Personen - eignet gesellschaftlichen, wirtschaftlichen oder politischen Tendenzen der Gegenwart ein sehr viel geringerer Grad an Spezifität: Im Vergleich mit den Fakten der Geschichte weisen sie keine derart klare raumzeitliche Referenz auf, und auch das Ausmaß ihrer Konventionalisierung, also ihrer allgemeinen Akzeptanz als Fakten für ein breites Publikum, ist oftmals deutlich geringer. Ein gewisser Grad an Spezifität ist jedoch nötig, um realweltliches Bezugsmaterial sinnvoll als Faktenmaterial bezeichnen zu können. Ein Element innerhalb einer fiktionalen Welt, das nicht auf Fakten der realen Welt, sondern lediglich auf allgemeine Realitätsannahmen verweist, kann nicht dem Weltvergleichsverhältnis der Kontrafaktik (oder Faktik) zugeschlagen werden, sondern rückt eher in den Bereich der Realistik oder Fantastik. Dass es sich bei den Einzeldaten der Geschichte um Fakten im engeren Sinne handelt, die entsprechend auch kontrafaktische Variationen erlauben, erscheint offenkundig (und dürfte einer der Hauptgründe dafür sein, dass die bisherige literaturwissenschaftliche Forschung zur Kontrafaktik sich vorwiegend mit der historischen Kontrafaktik auseinandergesetzt hat). ${ }^{1062}$ Wann allerdings eine gewisse Tendenz des öffentlichen Diskurses oder ein be-

1062 Siehe Kapitel 10. Historisches Erzählen als Kontrafaktik. 
stimmter politischer Trend, wie sie der Utopie/Dystopie für gewöhnlich zugrunde liegen, konkret genug ist, um als Fakt gelten zu können, lässt sich im Einzelfall nicht immer zweifelsfrei entscheiden.

Diese Problematik der Faktenspezifität innerhalb utopisch/dystopischer Texte kann genauer erläutert werden anhand einer kritischen Diskussion der Überlegungen Birte Christs zu den kontrafaktischen Potenzialen der feministisch-utopischen Literatur: Charlotte Perkins Gilmans Herland (1915) oder Sally Miller Gearharts The Wandergroud (1979) entwerfen reine Frauengesellschaft, in denen die Bewohnerinnen ein Leben frei von Sexismus, Gewinnstreben und Aggression führen, wie sie in patriarchalischen Gesellschaften vorherrschen. Aufgrund dieser kontrastierenden Verkehrung der Geschlechterstereotype klassifiziert Christ die genannten Texte als kontrafaktisch: „Both texts invert and contradict the male-defined logic that structures actual society down to the smallest detail and can thus be said to develop entire counterfactual worlds. “" ${ }^{1063}$ Man muss allerdings die Frage stellen, ob vermeintlich menschheitsübergreifende Geschlechtseigenschaften wirklich ein geeignetes Ausgangsmaterial für kontrafaktische Variationen bilden. Auch wenn man einmal davon absehen wollte, dass hier essentialistische Geschlechterklischees perpetuiert werden und die Frau primär über ihre Differenz zum Mann definiert wird (die Frau als mütterlich, emotional und friedlich, der Mann hingegen als kompetitiv, rational und aggressiv etc.), bliebe immer noch die Schwierigkeit bestehen, dass die unterstellte Geschlechtsnatur gerade kein historisch oder zeitlich lokalisierbares Einzelfaktum bildet, sondern vielmehr - zumindest in der Vorstellung der Autorinnen und ihrer intendierten Leserschaft - eine anthropologische Konstante. ${ }^{1064}$ Damit rücken die utopischen Gegenentwürfe von Texten wie Herland oder The Wanderground allerdings eher in Richtung der Fantastik als der Kontrafaktik: Hier wird nicht auf konkrete realweltliche Fakten Bezug genommen, deren Variation dann auch eine bestimmte, interpretatorisch relevante Kontextselektion des realweltlichen Faktenmaterials mit sich brächte. Stattdessen werden allgemeine Realitätsannahmen konterkariert.

Als Schwierigkeit für eine kontrafaktische Interpretation utopisch/dystopischer Texte tritt neben die beschriebene Problematik der Faktenspezifität auch

1063 Christ: "If I Were a Man”, S. 195.

1064 Eine kritisch-dekonstruktivistische Lektüre könnte freilich darauf aufmerksam machen, dass es sich bei der Vorstellung essentieller Geschlechtscharakteristika sowie bei deren konkreter Bestimmung sehr wohl um historisch und kulturell spezifische Wissensannahmen handelt, sodass ein Text über die ,eigentliche‘ und vermeintlich überzeitliche Natur der Geschlechter durchaus historisch konkretes ,Fakten`wissen transportieren würde. 
noch die Problematik der Konventionalisierung des Faktenmaterials: Jene Fakten der Gegenwart nämlich, auf die sich Utopien/Dystopien, aber häufig auch dokumentarische, satirische oder autofiktionale Texte beziehen, sind in der Durchschnittsenzyklopädie meist weniger fest verankert als das Wissen um zentrale Ereignisse der Geschichte. Entsprechend kann im Falle der Utopie/Dystopie nicht auf vergleichbar zuverlässige Weise mit einer kognitiven Aktualisierung des realweltlichen Faktenmaterials gerechnet werden wie im Falle von Alternate History-Erzählungen. Von besonderem interpretatorischen Interesse für Utopien/ Dystopien ist somit die Frage, welche national, historisch, ideologisch etc. spezifischen Publika hier jeweils adressiert werden und welches realweltliche Faktenmaterial dementsprechend als potenziell deutungsrelevant anzusehen ist.

Die Bestimmung des relevanten Faktenmaterials bildet im Falle von Utopien/ Dystopien also eine Herausforderung. Ein grundlegender Einwand gegen eine Konzeptualisierung von Utopien/Dystopien als Werke der Kontrafaktik ist damit jedoch nicht formuliert. Die Frage, welches realweltliche Faktenmaterial utopisch/dystopischen Erzählwelten zugrunde liegt und ob es sich dabei überhaupt um Faktenmaterial handelt, mag sich zwar nicht derart leicht beantworten lassen wie bei Werken der Alternate History. ${ }^{1065}$ Im Rahmen der Interpretation konkreter utopisch/dystopischer Texte können jedoch mitunter durchaus hochgradig plausible Entscheidungen bezüglich des relevanten Faktenmaterials getroffen werden. Welches Faktenmaterial in einem utopisch/dystopischen Werk genau alludiert wird - und ob sich diese Entscheidung überhaupt eindeutig treffen lässt -, hängt letztlich natürlich in hohem Maße vom jeweiligen Text ab.

Gerade die flexiblere, stärker von interpretatorischen Plausibilisierungsleistungen abhängige Faktenselektion, wie sie kontrafaktischen Deutungen utopisch/dystopischer Texte oftmals zugrunde liegt, kann im Einzelfall zu stark differierenden Interpretationsentscheidungen führen, über deren jeweilige Überzeugungskraft sich dann trefflich streiten lässt. Freilich bedeutet eine solche Polyvalenz für künstlerische Medien mitnichten ein Monitum. (Mit Leif Randts Roman Schimmernder Dunst über CobyCounty wird im Folgenden ein Text diskutiert, bei dem die interpretationsrelevante Faktenbasis vom Text gerade nicht eindeutig vorgegeben ist, sodass sich je nach Leserschaft unterschiedliche, auch ideologisch stark differierende Interpretationsvarianten ergeben.)

1065 Freilich ist auch für die historische Kontrafaktik (sowie für das historische Erzählen überhaupt) die Frage keineswegs unerheblich, welches Wissen von wem als historisches Faktenwissen akzeptiert wird. Vgl. McHale: Postmodernist Fiction, S. 87. 
Indem die vorliegende Studie die Potentiale der Kontrafaktik für das utopisch/dystopische Erzählen herausstellt, wird nicht nur eine isolierte Einsatzmöglichkeit der Kontrafaktik in einem beliebigen Genre benannt. Es soll vielmehr darauf aufmerksam gemacht werden, dass eine, wenn schon nicht notwendige Abhängigkeit, so doch ausgeprägte Affinität zwischen dem Genre der Utopie/Dystopie und der Referenzstruktur der Kontrafaktik besteht. Definiert man mit Wilhelm Voßkamp Utopien als „,anschaulich gemachte Entwürfe von positiven oder negativen Gegenbildern, die sich implizit oder explizit kritisch auf eine historische Wirklichkeit beziehen, in der sie entstanden sind“1066, so dürfte es kaum überraschen, dass die Anbindung utopisch/dystopischer Texte an die ,historische Wirklichkeit‘ häufig über Realitätsvariationen im Sinne der Kontrafaktik erfolgt. Die von Voßkamp als genredefinierend betrachtete Abhängigkeit von Gegenwart und Zukunft im Genre der Utopie/Dystopie kommt der transfiktionalen Referenzstruktur der Kontrafaktik in besonderem Maße entgegen.

Darüber hinaus legt auch die „kritisch[e]“ Dimension der „positiven oder negativen Gegenbilder[...]“ einen Einsatz der Kontrafaktik im Genre der Utopie/Dystopie nahe. Kritik an der Gegenwart kann durch einen fiktionalen Text nämlich nur dann vermittelt respektive vom Rezipienten erkannt werden, wenn die fiktionale Welt und die gesellschaftlich-politische Realität in irgendeiner Weise aufeinander bezogen sind, wenn also signifikante Strukturhomologien zwischen beiden vorliegen. ${ }^{1067}$ Im Genre der Utopie/Dystopie wird diese Strukturhomologie nun typischerweise nicht in Form eines Gegenwartsrealismus aufgebaut; stattdessen zeichnen sich utopisch/dystopische Erzählwelten durch ein amimetischfuturisches Setting aus, welches nichtsdestoweniger eine indirekte Verbindung zur politischen Gegenwart ihrer Entstehung und Rezeption unterhält. (Ein utopisch/dystopischer Text, der seine Verbindung zur politischen Gegenwart verlöre, könnte nicht mehr plausibel als utopisch/dystopischer Text bezeichnet werden; er ginge dann genremäßig eher in den Bereich der Science-Fiction oder Fantasy über.) Gerade dieser mit normativen Implikationen aufgeladene, indirekte Gegenwartsbezug ermöglicht es der Utopie/Dystopie, als Genre politischen Schreibens produktiv zu werden. Die Kontrafaktik bietet nun eine ideale Möglichkeit, einen solchen indirekten Gegenwartsbezug herzustellen, indem sie Elemente der fiktionalen Welt in Relation zu konkreten Fakten der realen Welt setzt. Der transfiktionale Weltvergleich der Kontrafaktik eröffnet immer auch die Möglichkeit einer normativen Kontrastierung von realer und fiktionaler Welt - und mithin auch

1066 Voßkamp: Utopie, S. 740.

1067 Vgl. Irsigler: World Gone Wrong, S. 180. 
die Möglichkeit politischen Schreibens. ${ }^{1068}$ Sowohl hinsichtlich ihrer genrekonstitutiv-indirekten Realitätsanbindung als auch hinsichtlich ihrer nicht weniger genrekonstitutiven politischen Dimension erweist sich die Utopie/ Dystopie somit als privilegiertes kontrafaktisches Genre, ja vermutlich als das große Einsatzgebiet der Kontrafaktik jenseits der Alternativgeschichte.

1068 Siehe Kapitel 9. Politische Kontrafaktik. 